\title{
Cosmological effects on the observed flux and fluence distributions of gamma-ray bursts: Are the most distant bursts in general the faintest ones?
}

\author{
A. Mészáros ${ }^{1}$, J. Řípa ${ }^{1}$, and F. Ryde ${ }^{2}$ \\ 1 Charles University, Faculty of Mathematics and Physics, Astronomical Institute, V Holešovičkách 2, 18000 Prague 8, \\ Czech Republic \\ e-mail:meszaros@cesnet.cz,ripa@sirrah.troja.mff.cuni.cz \\ 2 Department of Physics, Royal Institute of Technology, AlbaNova University Center, 10691 Stockholm, Sweden \\ e-mail: felix@particle.kth.se
}

Received 3 May 2010 / Accepted 19 January 2011

\section{ABSTRACT}

\begin{abstract}
Context. Several claims have been put forward that an essential fraction of long-duration BATSE gamma-ray bursts should lie at redshifts larger than 5. This point-of-view follows from the natural assumption that fainter objects should, on average, lie at larger redshifts. However, redshifts larger than 5 are rare for bursts observed by Swift, seemingly contradicting the BATSE estimates.

Aims. The purpose of this article is to clarify this contradiction.

Methods. We derive the cosmological relationships between the observed and emitted quantities, and we arrive at a prediction that can be tested on the ensembles of bursts with determined redshifts. This analysis is independent on the assumed cosmology, on the observational biases, as well as on any gamma-ray burst model. Four different samples are studied: 8 BATSE bursts with redshifts, 13 bursts with derived pseudo-redshifts, 134 Swift bursts with redshifts, and 6 Fermi bursts with redshifts.

Results. The controversy can be explained by the fact that apparently fainter bursts need not, in general, lie at large redshifts. Such a behaviour is possible, when the luminosities (or emitted energies) in a sample of bursts increase more than the dimming of the observed values with redshift. In such a case $\mathrm{d} P(z) / \mathrm{d} z>0$ can hold, where $P(z)$ is either the peak-flux or the fluence. All four different samples of the long bursts suggest that this is really the case. This also means that the hundreds of faint, long-duration BATSE bursts need not lie at high redshifts, and that the observed redshift distribution of long Swift bursts might actually represent the actual distribution.
\end{abstract}

Key words. gamma-ray burst: general - cosmology: miscellaneous

\section{Introduction}

Until the last years, the redshift distribution of gamma-ray bursts (GRBs) has only been poorly known. For example, the Burst And Transient Source Experiment (BATSE) instrument on Compton Gamma Ray Observatory detected around 2700 GRBs $^{1}$, but only a few of these have directly measured redshifts from the afterglow (AG) observations (Schaefer 2003; Piran 2004). During the last couple of years the situation has improved, mainly due to the observations made by the Swift satellite $^{2}$. There are already dozens of directly determined redshifts (Mészáros 2006). Nevertheless, this sample is only a small fraction of the, in total, thousands of detected bursts.

Beside the direct determination of redshifts from the AGs, there are several indirect methods, which utilize the gamma-ray data alone. In essence, there are two different methods which provide such determinations. The first one makes only statistical estimations; the fraction of bursts in a given redshift interval is deduced. The second one determines an actual value of the redshift for a given GRB ("pseudo-redshift")

Already at the early 1990's, that is, far before the observation of any $\mathrm{AG}$, and when even the cosmological origin was in doubt,

\footnotetext{
${ }^{1}$ http://www.batse.msfc.nasa.gov/batse/grb/catalog/

${ }^{2}$ http://swift.gsfc.nasa.gov/docs/swift/swiftsc.html
}

there were estimations made in the sense of the first method (see, e.g., Paczyński 1992, and the references therein). In Mészáros \& Mészáros (1996) a statistical study indicated that a fraction of GRBs should be at very large redshifts (up to $z \simeq 20$ ). In addition, there was no evidence for the termination of the increase of the numbers of GRBs for $z>5$ (see also Mészáros \& Mészáros 1995; Horváth et al. 1996, and Reichart \& Mészáros 1997). In other words, an essential fraction (a few or a few tens of \%) of GRBs should be in the redshift interval $5<z<20$. Again using this type of estimation, Lin et al. (2004) claims that even the majority of bursts should be at these high redshifts.

The estimations of the pseudo-redshifts in the sense of the second method are more recent. Ramirez-Ruiz \& Fenimore (2000), Reichart et al. (2001), Schaefer et al. (2001) and Lloyd-Ronning et al. (2002) developed a method allowing to obtain from the so-called variability the intrinsic luminosity of a GRB, and then from the measured flux its redshift. The redshifts of hundred of bursts were obtained by this method. Nevertheless, the obtained pseudo-redshifts are in doubt, because there is an $(1+z)$ factor error in the cosmological formulas (Norris 2004; Band et al. 2004). Other authors also query these redshifts (e.g., Guidorzi et al. 2005). Norris et al. (2000) found another relation between the spectral lag and the luminosity. This method seems to be a better one (Schaefer et al. 2001; Norris 2002; Ryde et al. 2005), and led to the estimation of $\sim 1200$ burst 
redshifts. Remarkably, again, an essential fraction of long GRBs should have $z>5$, and the redshift distribution should peak at $z \sim 10$. A continuation of this method (Band et al. 2004; Norris 2004), which corrected the $(1+z)$ error in Norris (2002), gave smaller redshifts on average, but the percentage of long GRBs at $z>5$ remains open. Bagoly et al. (2003) developed a different method allowing to obtain the redshifts from the gamma spectra for 343 bright GRBs. Due to the two-fold character of the estimation, the fraction of GRBs at $z>5$ remains further open. No doubt has yet emerged concerning this method. Atteia (2003) also proposed a method in a similar sense for bright bursts. Other methods of such estimations also exist (Amati et al. 2002; Ghirlanda et al. 2005). These pseudo-redshift estimations (for a survey see, e.g. Sect. 2.6 of Mészáros 2006) give the results that even bright GRBs should be at redshifts $z \sim(1-5)$. For faint bursts in the BATSE data set (i.e. for GRBs with peakfluxes smaller than $\simeq 1$ photon/ $\left(\mathrm{cm}^{2} \mathrm{~s}\right)$ ) one hardly can have good pseudo-redshift estimations, but on average they are expected to be at larger redshifts, using the natural assumption that these bursts are observationally fainter due to their larger distances. Hence, it is remarkable that all these pseudo-redshift estimations also supports the expectation, similarly to the results of the first method, that an essential fraction of GRBs is at very high redshifts.

Contrary to these estimations for the BATSE data set, only five long bursts with $z>5$ have yet been detected from direct redshift measurements from AGs using more recent satellites. In addition, the majority of measured $z$-s are around $z=2-3$, and there is a clear decreasing tendency towards the larger redshifts ${ }^{3}$. In other words, the redshifts of GRBs detected by the Swift satellite do not support the BATSE redshift estimations; the redshifts of GRBs detected by the non-Swift satellites are on average even at smaller redshifts (Bagoly et al. 2006).

This can be interpreted in two essentially different ways. The first possibility is that a large fraction (a few tens of \% or even the majority) of GRBs are at very high redshifts (at $z>5$ or so). In such case these bursts should mainly be seen only in the gamma-ray band due to some observational selection (Lamb \& Reichart 2000). The second possibility is that the AG data reflect the true distribution of bursts at high redshifts, and bursts at $z>5$ are really very rare. In this case, however, there has to be something wrong in the estimations of redshifts from the gamma-ray data alone. Since observational selections for AG detections of bursts at $z>5$ cannot be excluded (Lamb \& Reichart 2000), the first point-of-view could be also quite realistic.

The purpose of this paper is to point out some statistical properties of the GRBs, which may support the second possibility. Section 2 discusses these properties theoretically. In Sects. 3 and 4 we discuss the impact of such a behaviour on several observed burst samples. Section 5 summarizes the results of paper.

\section{Theoretical considerations}

\subsection{The general consideration}

Using the standard cosmological theory and some simple statistical considerations, we will now show that, under some conditions, apparently fainter bursts might very well be at smaller redshifts compared to the brighter ones.

As shown by Mészáros \& Mészáros (1995), if there are given two photon-energies $E_{1}$ and $E_{2}$, where $E_{1}<E_{2}$, then the

\footnotetext{
${ }^{3}$ http://www.mpe.mpg.de/ jcg/grbgen.html
}

flux $F$ (in units photons/ $\left(\mathrm{cm}^{2} \mathrm{~s}\right)$ ) of the photons with energies $E_{1} \leq E \leq E_{2}$ detected from a GRB having a redshift $z$ is given by

$$
F_{\mathrm{ph}}(z)=\frac{(1+z) \tilde{L}_{\mathrm{ph}}(z)}{4 \pi d_{1}(z)^{2}}=\frac{\tilde{L}_{\mathrm{ph}}(z)}{4 \pi d_{\mathrm{M}}(z)^{2}(1+z)},
$$

where $\tilde{L}_{\mathrm{ph}}(z)$ is the isotropic luminosity of a GRB (in units photons/s) between the energy range $E_{1}(1+z) \leq E \leq E_{2}(1+z)$, and $d_{1}(z)$ is the luminosity distance of the source. The reason of the notation $\tilde{L}(z)$, instead of the simple $L(z)$, is that $L(z)$ should mean the luminosity from $E_{1} \leq E \leq E_{2}$ (Mészáros et al. 2006). One has $d_{1}(z)=(1+z) d_{\mathrm{M}}(z)$, where $d_{\mathrm{M}}(z)$ is the proper motion distance of the GRB, given by the standard cosmological formula (Carroll et al. 1992), and depends on the cosmological parameters $H_{0}$ (Hubble-constant), $\Omega_{\mathrm{M}}$ (the ratio of the matter density and the critical density), and $\Omega_{\Lambda}=3 \lambda c^{2} /\left(3 H_{0}^{2}\right)(\lambda$ is the cosmological constant, $c$ is the velocity of light). In energy units one may write $F_{\mathrm{en}}(z)=\bar{E} F_{\mathrm{ph}}(z)$ and $\tilde{L}_{\mathrm{en}}(z)=(1+z) \bar{E} \tilde{L}_{\mathrm{ph}}(z)$, where $\bar{E}\left(E_{1}<\bar{E}<E_{2}\right)$ is a typical photon energy ensuring that the flux $F_{\text {en }}(z)$ has the dimension erg $/\left(\mathrm{cm}^{2} \mathrm{~s}\right) . \tilde{L}_{\mathrm{en}}(z)$ in $\mathrm{erg} / \mathrm{s}$ unit gives the luminosity in the interval $E_{1}(1+z) \leq E \leq E_{2}(1+z)$. Except for an $(1+z)$ factor the situation is the same, when considering the fluence. Hence, in the general case, we have

$P(z)=\frac{(1+z)^{N} \tilde{L}(z)}{4 \pi d_{1}(z)^{2}}$,

where $P(z)$ is either the fluence or the flux, and $\tilde{L}(z)$ is either the emitted isotropic total number of photons, or the isotropic total emitted energy or the luminosity. The following values of $N$ can be used: $N=0$ if the flux is taken in energy units $\mathrm{erg} /\left(\mathrm{cm}^{2} \mathrm{~s}\right)$ and $\tilde{L}$ is the luminosity with dimension erg/s; $N=1$ if either the flux and luminosity are in photon units, or the fluence in energy units and the total energy are taken; $N=2$ if the total number of photons are considered. All this means that for a given GRB - for which redshift, flux and fluence are measured - Eq. (2) defines immediately $\tilde{L}(z)$, which is then either the isotropic total emitted energy or the luminosity in the interval $E_{1}(1+z) \leq E \leq E_{2}(1+z)$. Hence, $\tilde{L}(z)$ is from the range $E_{1}(1+z) \leq E \leq E_{2}(1+z)$ and not from $E_{1} \leq E \leq E_{2}$.

Let us have a measured value of $P(z)$. Fixing this $P(z)$ Eq. (2) defines a functional relationship between the redshift $z$ and $\tilde{L}(z)$. For its transformation into the real intrinsic luminosities $L(z)$ the beaming must be taken into account as well (Lee et al. 2000; Ryde et al. 2005; Bagoly \& Veres 2009a,b; Petrosian et al. 2009; Butler et al. 2010). Additionally, we need to study the dependence of the obtained $\tilde{L}(z)$ on $z$, and to determine the real luminosities $L(z)$ by the K-corrections (Mészáros 2006). It is not the aim of this paper to solve the transformation of $\tilde{L}(z)$ into $L(z)$. The purpose of this paper is to study the functional relationship among $P(z), z$ and $\tilde{L}(z)$.

Using the proper motion distance $d_{\mathrm{M}}(z)$ Eq. (2) can be reordered as

$4 \pi d_{\mathrm{M}}(z)^{2}(1+z)^{2-N} P(z)=\tilde{L}(z)$.

The proper motion distance $d_{\mathrm{M}}(z)$ is bounded as $z \rightarrow \infty$ (Weinberg 1972, Chap. 14.4.). This finiteness of the proper motion distance is fulfilled for any $H_{0}, \Omega_{\mathrm{M}}$ and $\Omega_{\Lambda}$. Hence, $\tilde{L}(z)$ is a monotonically increasing function of the redshift along with $(1+z)^{2-N}$ for the fixed $P(z)=P_{0}$ and for the given value of $N \leq 1$. It means $z_{1}<z_{2}$ implies $\tilde{L}\left(z_{1}\right)<\tilde{L}\left(z_{2}\right)$. Expressing this result in other words: the more distant and brighter sources may give the same observed value of $P_{0}$. Now, if a source at $z_{2}$ has a $\tilde{L}>\tilde{L}\left(z_{2}\right)$, its observed value $P_{\text {obs }}^{\prime}$ will have $P_{\text {obs }}^{\prime}>P_{0}$, i.e. it becomes apparently brighter despite its greater redshift than that of 
A. Mészáros et al.: Cosmological and gamma-ray bursts
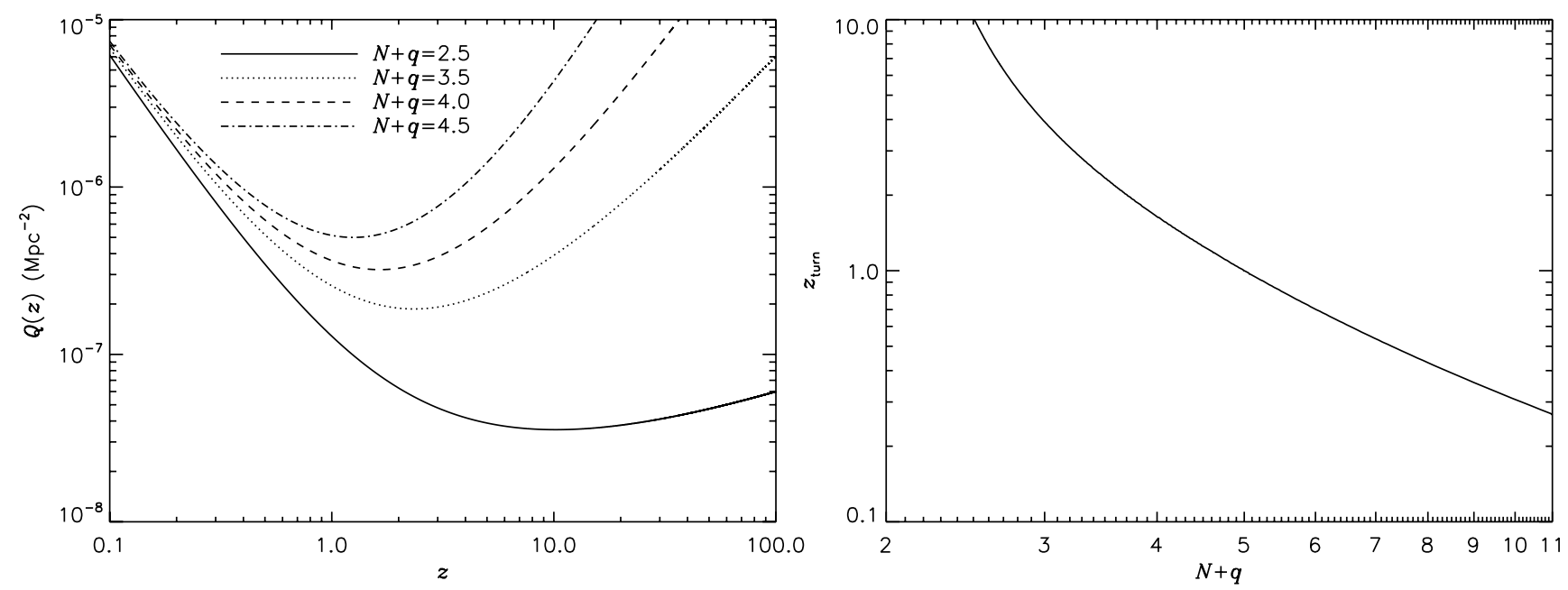

Fig. 1. Left panel: function $Q(z)$ for $\Omega_{\mathrm{M}}=0.27$ and $\Omega_{\Lambda}=0.73$. Right panel: dependence of $z_{\text {turn }}$ on $N+q$ for $\Omega_{\mathrm{M}}=0.27, \Omega_{\Lambda}=0.73$.

the source at $z_{1}$. The probability of the occurrence this kind of inversion depends on $f(\tilde{L} \mid z)$, on the conditional probability density of $\tilde{L}$ assuming $z$ is given, and on the spatial density of the objects.

It is obvious from Eq. (3) that for the increasing $z$ the $\tilde{L}(z)$ is also increasing. This effect gives a bias (Lee et al. 2000; Bagoly \& Veres 2009a,b; Petrosian et al. 2009; Butler et al. 2010) towards the higher $\tilde{L}(z)$ values among GRBs observed above a given detection threshold. (These questions are discussed in detail mainly by Petrosian et al. 2009.) There can be also that $\tilde{L}(z)$ is increasing with $z$ due to the metallicity evolution (Wolf \& Podsiadlowski 2007). There can be also an intrinsic evolution of the $L(z)$ itself in the energy range $\left[E_{1}, E_{2}\right]$. Hence, keeping all this in mind, $\tilde{L}(z)$ can well be increasing on $z$, and the inverse behaviour can also occur.

\subsection{A special assumption}

Assume now that we have $\tilde{L}(z) \propto(1+z)^{q}$, where $q$ is a real number, and this relation holds for any $0<z<\infty$. This means that it holds $\tilde{L}(z)=\tilde{L}_{0}(1+z)^{q}$, where $\tilde{L}(z=0)=\tilde{L}_{0}$. Of course, this special assumption is a loss of generality, because $\tilde{L}(z)$ can be really a function of $z$, but in general it need not have this special form. In addition, to calculate $\tilde{L}(z)$ the cosmological parameters must be specified, which is a further loss of generality. Nevertheless, if this special assumption is taken, the inverse behavior may well be demonstrated.

If $(N+q)>2$, then one has a highly interesting mathematical behaviour of the function $P(z)$ (Eq. (2)). For $z \ll 0.1, P(z)$ decreases as $z^{-2}$, that is, larger redshifts gives smaller fluxes or fluences as expected. However, after some $z=z_{\text {turn }}$ this behavior must change, because as $z$ tends to $\infty$, the function $(1+z)^{N} \tilde{L} / d_{1}^{2}$ tends to infinity following $\propto(1+z)^{N+q-2}$. In other words, for $z>z_{\text {turn }}$ as redshift increases, the measured $P(z)$ will also increase. Equivalently stated, "fainter bursts are closer to us". The possibility of this "inverse" behaviour is quite remarkable. It is important to note that the existence of a $z_{\text {turn }}$ is exclusively determined by the value $q$, and the necessary and sufficient condition for it is given by the inequality $(N+q)>2$. For the existence of a $z_{\text {turn }}$ the values $H_{0}, \Omega_{\mathrm{M}}$ and $\Omega_{\Lambda}$ are unimportant. The value of $z_{\text {turn }}$ can vary depending on the choice of the $\Omega$ parameters, but, however, its existence or non-existence is unaffected.

Moreover, the value of $z_{\text {turn }}$ itself is independent on the Hubble-constant $H_{0}$. This can be seen as follows. To find $z_{\text {turn }}$ one must simply search for the minimum of the function
$Q(z)=(1+z)^{N+q} / d_{1}(z)^{2}$, that is, when $\mathrm{d} Q(z) / \mathrm{d} z=0$. But, trivially, $Q(z)$ and $Q(z) / H_{0}^{2}$ have the same minimum.

The solution of the equation $\mathrm{d} Q(z) / \mathrm{d} z=0$ must be found numerically for the general case of Omega parameters. The left panel of Fig. 1 shows the function $Q(z)$ for $\Omega_{\mathrm{M}}=0.27$ and $\Omega_{\Lambda}=$ 0.73 . For $\Omega_{\Lambda}=0$ it can be found analytically, because $d_{\mathrm{M}}(z)$ is then an analytical function. For $\Omega_{\mathrm{M}}=1$ and $\Omega_{\Lambda}=0$ the condition $\mathrm{d} Q(z) / \mathrm{d} z=0$ is easily solvable. For this special case one has to search for the minimum of function

$$
\frac{(1+z)^{N+q-2}}{\left(1-(1+z)^{-1 / 2}\right)^{2}}
$$

because here $d_{\mathrm{M}}=\left(2 c / H_{0}\right) /\left(1-(1+z)^{-1 / 2}\right)$. Then one has

$z_{\text {turn }}=\left(\frac{N+q-1}{N+q-2}\right)^{2}-1$.

\section{The samples}

\subsection{The choice of burst samples}

The frequency of the occurrence $\tilde{L}\left(z_{1}\right)<\tilde{L}\left(z_{2}\right)$ at $z_{1}<z_{2}$, but for their observed values $P\left(z_{1}\right)<P\left(z_{2}\right)$, i.e. the more distant GRB is apparently brighter for the observer, can be verified on a sample for which there are well-defined redshifts, as well as measured peak-fluxes and/or fluences. At a given redshift the luminosity $\tilde{L}(z)$ is a stochastic variable and starting from Eq. (3) one can get the probability for $P_{\mathrm{obs}}^{\prime}>P(z)$, assuming that $f(\tilde{L} \mid z)$ is given.

There are two different subgroups of GRBs, which can be denoted as "short-" and "long-"duration bursts (Norris et al. 2001; Balázs et al. 2003; Mészáros et al. 2006; Zhang et al. 2009). In addition, the existence of additional subgroups cannot be excluded (Horváth 1998, 2002; Hakkila et al. 2003; Borgonovo 2004; Varga et al. 2005; Horváth et al. 2008, 2009; Vavrek et al. 2008). The first direct redshift for a long (short) GRBs was determined in 1997 (2005) (for a detailed survey see, e.g., Piran 2004; and Mészáros 2006). The few redshifts measured for short bursts are on average small (Mészáros et al. 2009), which motivates us to concentrate on long-duration bursts only in this study.

Since we try to obtain consequences of the GRBs' redshifts also in the BATSE Catalog, we should obviously study the BATSE sample. But only a few of these bursts have directly measured redshifts from afterglow data. Therefore we will 

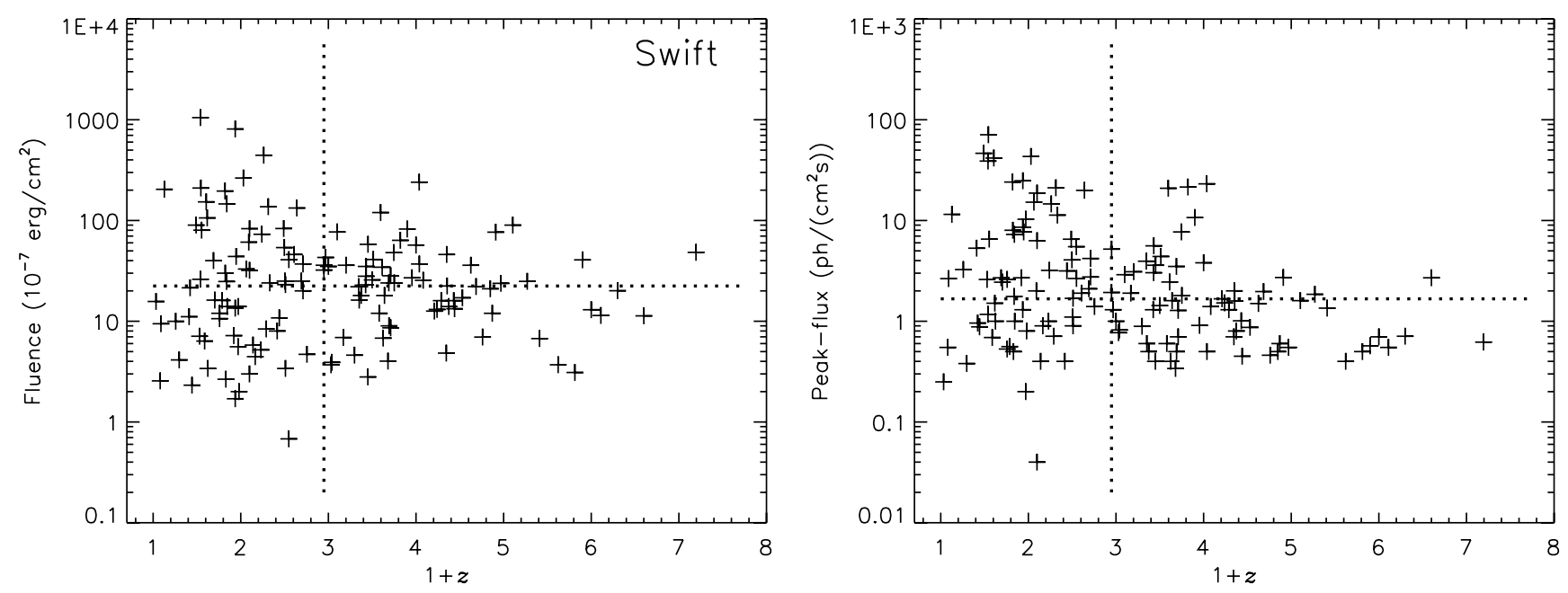

Fig. 2. Distribution of the fluences (left panel) and peak-fluxes (right panel) of the Swift GRBs with known redshifts. The medians separate the area into four quadrants. The objects in the upper right quadrant are brighter and have larger redshifts than the that of GRBs in the lower left quadrant.
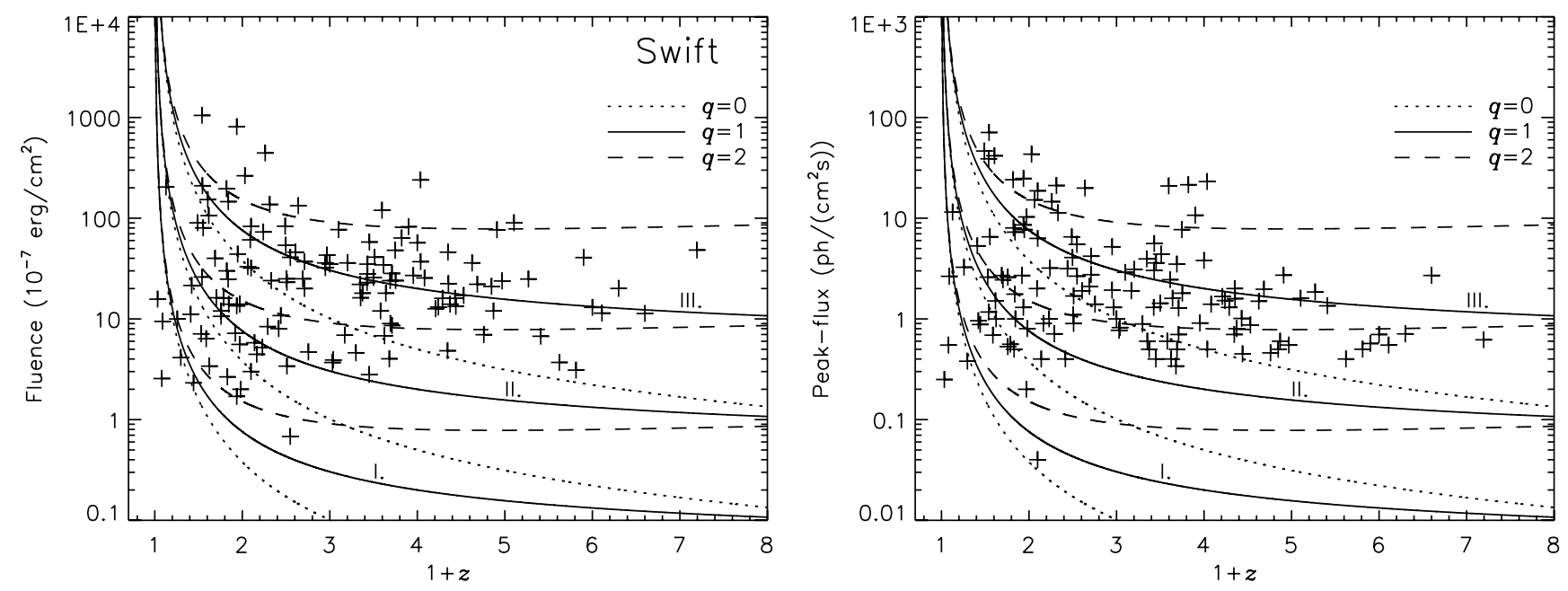

Fig. 3. Distribution of the fluences (left panel) and peak-fluxes (right panel) of the Swift GRBs with known redshifts. On the left panel the curves denote the values of fluences for $\tilde{E}_{\text {iso }}=\tilde{E}_{0}(1+z)^{q}$ (the three constant $\tilde{E}_{\mathrm{o}}$ are in units $10^{51} \mathrm{erg}$ : I. 0.1 ; II. 1.0; III. 10.0). On the right panel the curves

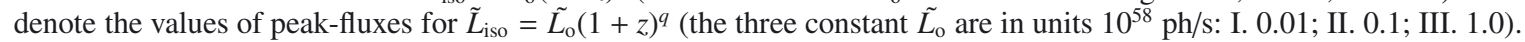

also try to obtain conclusions from a sample that uses the socalled "pseudo-redshifts", i.e. the redshifts estimated exclusively from the gamma photon measurements alone. But for them one should keep in mind that they can be uncertain. Thus, the best is to compare the BATSE samples with other samples of long GRBs. The redshifts of GRBs detected by Swift satellite - obtained from afterglow data - can well serve for this comparison. On the other hand, the redshifts of GRBs - detected by other satellites (BeppoSAX, HETE-2, INTEGRAL) - are not good for our purpose, since they are strongly biased with selection effects (Lee et al. 2000; Bagoly et al. 2006; Butler et al. 2010), and represent only the brightest bursts. All this means that we will discuss four samples here: BATSE GRBs with known redshifts, BATSE GRBs with pseudo-redshifts, the Swift sample and the Fermi sample. We will try to show the occurrence of the inverse behaviour, first, without the special assumptions of Sect. 2.2., and, second, using this subsection.

\subsection{Swift GRBs and the inversion in this sample}

In the period of 20 November 2004-30 April 2010 the Swift database gives a sample of 134 bursts with well determined redshifts from the afterglows together with BAT fluences and peak-fluxes in the energy range of $15-150 \mathrm{keV}$. To abandon the short bursts only those with $T_{90} /(1+z)>2 \mathrm{~s}$ were taken. They are collected in Table 1.

The effect of inversion can be demonstrated by the scatter plots of the $[\log$ fluence; $z]$ and $[\log$ peak-flux; $z$ ] planes as it can be seen in Fig. 2. To demonstrate the effect of inversion we marked the medians of the fluence and peak-flux with horizontal and that of the redshift with vertical dashed lines. The medians split the plotting area into four quadrants. It is easy to see that GRBs in the upper right quadrant are apparently brighter than those in the lower left one, although, their redshifts are larger. It is worth mentioning that the GRB having the greatest redshift in the sample has higher fluence than $50 \%$ of all the items in the sample.

Using the special assumption of Sect. 2.2. the effect of inversion may be illustrated in the Swift sample distinctly also as follows. In Fig. 3 the fluences and peak-fluxes are typified against the redshifts. Be the luminosity distances calculated for $H_{0}=$ $71 \mathrm{~km} /(\mathrm{s} \mathrm{Mpc}), \Omega_{\mathrm{M}}=0.27$ and $\Omega_{\Lambda}=0.73$. Then the total emitted energy $\tilde{E}_{\text {iso }}$ and the peak-luminosity $\tilde{L}_{\text {iso }}$ can be calculated using Eq. (2) with $N=1$. In the figure the curves of fluences and 
A. Mészáros et al.: Cosmological and gamma-ray bursts

Table 1. Swift sample with known redshifts.

\begin{tabular}{|c|c|c|c|c|c|c|}
\hline GRB & $\begin{array}{c}\text { Fluence } \\
10^{-7} \mathrm{erg} / \mathrm{cm}^{2}\end{array}$ & $\begin{array}{l}\text { Peak-flux } \\
\mathrm{ph} /\left(\mathrm{cm}^{2} \mathrm{~s}\right)\end{array}$ & $z$ & $\begin{array}{c}d_{1} \\
\mathrm{Gpc}\end{array}$ & $\begin{array}{c}\tilde{E}_{\text {iso }} \\
10^{51} \text { erg }\end{array}$ & $\begin{array}{c}\tilde{L}_{\text {iso }} \\
10^{58} \mathrm{ph} / \mathrm{s}\end{array}$ \\
\hline 050126 & 8.38 & 0.71 & 1.29 & 9.12 & $3.6 \mathrm{E}+0$ & $3.1 \mathrm{E}-1$ \\
\hline 050223 & 6.36 & 0.69 & 0.588 & 3.44 & $5.7 \mathrm{E}-1$ & $6.1 \mathrm{E}-2$ \\
\hline 050315 & 32.2 & 1.93 & 1.949 & 15.24 & $3.0 \mathrm{E}+1$ & $1.8 \mathrm{E}+0$ \\
\hline 050318 & 10.8 & 3.16 & 1.44 & 10.46 & $5.8 \mathrm{E}+0$ & $1.7 \mathrm{E}+0$ \\
\hline 050319 & 13.1 & 1.52 & 3.24 & 28.37 & $3.0 \mathrm{E}+1$ & $3.5 \mathrm{E}+0$ \\
\hline 050401 & 82.2 & 10.7 & 2.9 & 24.81 & $1.6 \mathrm{E}+2$ & $2.0 \mathrm{E}+1$ \\
\hline 050505 & 24.9 & 1.85 & 4.27 & 39.49 & $8.8 \mathrm{E}+1$ & $6.6 \mathrm{E}+0$ \\
\hline $050525 \mathrm{~A}$ & 153 & 41.7 & 0.606 & 3.57 & $1.5 \mathrm{E}+1$ & $4.0 \mathrm{E}+0$ \\
\hline 050603 & 63.6 & 21.5 & 2.821 & 23.99 & $1.1 \mathrm{E}+2$ & $3.9 \mathrm{E}+1$ \\
\hline 050724 & 9.98 & 3.26 & 0.258 & 1.29 & $1.6 \mathrm{E}-1$ & $5.2 \mathrm{E}-2$ \\
\hline 050730 & 23.8 & 0.55 & 3.969 & 36.20 & $7.5 \mathrm{E}+1$ & $1.7 \mathrm{E}+0$ \\
\hline 050802 & 20 & 2.75 & 1.71 & 12.96 & $1.5 \mathrm{E}+1$ & $2.0 \mathrm{E}+0$ \\
\hline 050803 & 21.5 & 0.96 & 0.422 & 2.30 & $9.6 \mathrm{E}-1$ & $4.3 E-2$ \\
\hline 050814 & 20.1 & 0.71 & 5.3 & 50.99 & $9.9 \mathrm{E}+1$ & $3.5 \mathrm{E}+0$ \\
\hline 050820A & 34.4 & 2.45 & 2.613 & 21.85 & $5.4 \mathrm{E}+1$ & $3.9 \mathrm{E}+0$ \\
\hline 050824 & 2.66 & 0.5 & 0.83 & 5.26 & $4.8 \mathrm{E}-1$ & $9.0 \mathrm{E}-2$ \\
\hline 050826 & 4.13 & 0.38 & 0.297 & 1.52 & $8.8 \mathrm{E}-2$ & $8.1 \mathrm{E}-3$ \\
\hline 050904 & 48.3 & 0.62 & 6.195 & 61.22 & $3.0 \mathrm{E}+2$ & $3.9 \mathrm{E}+0$ \\
\hline 050908 & 4.83 & 0.7 & 3.346 & 29.49 & $1.2 \mathrm{E}+1$ & $1.7 \mathrm{E}+0$ \\
\hline 051016B & 1.7 & 1.3 & 0.936 & 6.11 & $3.9 \mathrm{E}-1$ & $3.0 \mathrm{E}-1$ \\
\hline 051109A & 22 & 3.94 & 2.346 & 19.15 & $2.9 \mathrm{E}+1$ & $5.2 \mathrm{E}+0$ \\
\hline 051109B & 2.56 & 0.55 & 0.08 & 0.36 & $3.7 \mathrm{E}-3$ & $7.8 \mathrm{E}-4$ \\
\hline 051111 & 40.8 & 2.66 & 1.549 & 11.46 & $2.5 \mathrm{E}+1$ & $1.6 \mathrm{E}+0$ \\
\hline 060108 & 3.69 & 0.77 & 2.03 & 16.03 & $3.7 \mathrm{E}+0$ & $7.8 \mathrm{E}-1$ \\
\hline 060115 & 17.1 & 0.87 & 3.53 & 31.45 & $4.5 \mathrm{E}+1$ & $2.3 \mathrm{E}+0$ \\
\hline 060123 & 3 & 0.04 & 1.099 & 7.47 & $9.5 \mathrm{E}-1$ & $1.3 \mathrm{E}-2$ \\
\hline 060124 & 4.61 & 0.89 & 2.298 & 18.67 & $5.8 \mathrm{E}+0$ & $1.1 \mathrm{E}+0$ \\
\hline 060210 & 76.6 & 2.72 & 3.91 & 35.55 & $2.4 \mathrm{E}+2$ & $8.4 \mathrm{E}+0$ \\
\hline 060218 & 15.7 & 0.25 & 0.033 & 0.14 & $3.7 \mathrm{E}-3$ & $5.9 \mathrm{E}-5$ \\
\hline 060223A & 6.73 & 1.35 & 4.41 & 41.03 & $2.5 \mathrm{E}+1$ & $5.0 \mathrm{E}+0$ \\
\hline 060418 & 83.3 & 6.52 & 1.49 & 10.91 & $4.8 \mathrm{E}+1$ & $3.7 \mathrm{E}+0$ \\
\hline 060502A & 23.1 & 1.69 & 1.51 & 11.10 & $1.4 \mathrm{E}+1$ & $9.9 \mathrm{E}-1$ \\
\hline 060505 & 9.44 & 2.65 & 0.089 & 0.40 & $1.7 \mathrm{E}-2$ & $4.7 \mathrm{E}-3$ \\
\hline 060510B & 40.7 & 0.57 & 4.9 & 46.49 & $1.8 \mathrm{E}+2$ & $2.5 \mathrm{E}+0$ \\
\hline 060512 & 2.32 & 0.88 & 0.443 & 2.44 & $1.1 \mathrm{E}-1$ & $4.3 \mathrm{E}-2$ \\
\hline 060522 & 11.4 & 0.55 & 5.11 & 48.85 & $5.3 \mathrm{E}+1$ & $2.6 \mathrm{E}+0$ \\
\hline 060526 & 12.6 & 1.67 & 3.21 & 28.05 & $2.8 \mathrm{E}+1$ & $3.7 \mathrm{E}+0$ \\
\hline $060602 \mathrm{~A}$ & 16.1 & 0.56 & 0.787 & 4.92 & $2.6 \mathrm{E}+0$ & $9.1 \mathrm{E}-2$ \\
\hline 060604 & 4.02 & 0.34 & 2.68 & 22.54 & $6.6 \mathrm{E}+0$ & $5.6 \mathrm{E}-1$ \\
\hline 060605 & 6.97 & 0.46 & 3.76 & 33.93 & $2.0 \mathrm{E}+1$ & $1.3 \mathrm{E}+0$ \\
\hline 060607A & 25.5 & 1.4 & 3.082 & 26.71 & $5.3 \mathrm{E}+1$ & $2.9 \mathrm{E}+0$ \\
\hline 060614 & 204 & 11.5 & 0.128 & 0.59 & $7.6 E-1$ & $4.3 E-2$ \\
\hline 060707 & 16 & 1.01 & 3.43 & 30.39 & $4.0 \mathrm{E}+1$ & $2.5 \mathrm{E}+0$ \\
\hline 060714 & 28.3 & 1.28 & 2.71 & 22.84 & $4.8 \mathrm{E}+1$ & $2.2 \mathrm{E}+0$ \\
\hline 060729 & 26.1 & 1.17 & 0.54 & 3.10 & $1.9 \mathrm{E}+0$ & $8.7 \mathrm{E}-2$ \\
\hline 060814 & 146 & 7.27 & 0.84 & 5.34 & $2.7 \mathrm{E}+1$ & $1.3 \mathrm{E}+0$ \\
\hline 060904B & 16.2 & 2.44 & 0.703 & 4.28 & $2.1 \mathrm{E}+0$ & $3.1 \mathrm{E}-1$ \\
\hline 060906 & 22.1 & 1.97 & 3.685 & 33.12 & $6.2 \mathrm{E}+1$ & $5.5 \mathrm{E}+0$ \\
\hline 060908 & 28 & 3.03 & 2.43 & 20.00 & $3.9 \mathrm{E}+1$ & $4.2 \mathrm{E}+0$ \\
\hline 060912 & 13.5 & 8.58 & 0.937 & 6.12 & $3.1 \mathrm{E}+0$ & $2.0 \mathrm{E}+0$ \\
\hline 060927 & 11.3 & 2.7 & 5.6 & 54.40 & $6.1 \mathrm{E}+1$ & $1.4 \mathrm{E}+1$ \\
\hline 061007 & 444 & 14.6 & 1.262 & 8.87 & $1.8 \mathrm{E}+2$ & $6.1 \mathrm{E}+0$ \\
\hline 061110A & 10.6 & 0.53 & 0.758 & 4.70 & $1.6 \mathrm{E}+0$ & $8.0 \mathrm{E}-2$ \\
\hline 061110B & 13.3 & 0.45 & 3.44 & 30.49 & $3.3 \mathrm{E}+1$ & $1.1 \mathrm{E}+0$ \\
\hline 061121 & 137 & 21.1 & 1.314 & 9.33 & $6.2 \mathrm{E}+1$ & $9.5 \mathrm{E}+0$ \\
\hline 061210 & 11.1 & 5.31 & 0.41 & 2.22 & $4.7 \mathrm{E}-1$ & $2.2 \mathrm{E}-1$ \\
\hline 061222B & 22.4 & 1.59 & 3.355 & 29.59 & $5.4 \mathrm{E}+1$ & $3.8 \mathrm{E}+0$ \\
\hline 070110 & 16.2 & 0.6 & 2.352 & 19.21 & $2.1 \mathrm{E}+1$ & $7.9 \mathrm{E}-1$ \\
\hline 070208 & 4.45 & 0.9 & 1.165 & 8.03 & $1.6 \mathrm{E}+0$ & $3.2 \mathrm{E}-1$ \\
\hline 070306 & 53.8 & 4.07 & 1.497 & 10.98 & $3.1 \mathrm{E}+1$ & $2.4 \mathrm{E}+0$ \\
\hline 070318 & 24.8 & 1.76 & 0.838 & 5.32 & $4.6 \mathrm{E}+0$ & $3.2 \mathrm{E}-1$ \\
\hline 070411 & 27 & 0.91 & 2.954 & 25.37 & $5.3 \mathrm{E}+1$ & $1.8 \mathrm{E}+0$ \\
\hline 070419A & 5.58 & 0.2 & 0.97 & 6.39 & $1.4 \mathrm{E}+0$ & $5.0 \mathrm{E}-2$ \\
\hline 070508 & 196 & 24.1 & 0.82 & 5.18 & $3.5 \mathrm{E}+1$ & $4.3 \mathrm{E}+0$ \\
\hline 070521 & 80.1 & 6.53 & 0.553 & 3.19 & $6.3 \mathrm{E}+0$ & $5.1 \mathrm{E}-1$ \\
\hline
\end{tabular}


Table 1. continued.

\begin{tabular}{|c|c|c|c|c|c|c|}
\hline GRB & $\begin{array}{c}\text { Fluence } \\
10^{-7} \mathrm{erg} / \mathrm{cm}^{2}\end{array}$ & $\begin{array}{l}\text { Peak-flux } \\
\mathrm{ph} /\left(\mathrm{cm}^{2} \mathrm{~s}\right)\end{array}$ & $z$ & $\begin{array}{c}d_{1} \\
\mathrm{Gpc}\end{array}$ & $\begin{array}{c}\tilde{E}_{\text {iso }} \\
10^{51} \text { erg }\end{array}$ & $\begin{array}{c}\tilde{L}_{\text {iso }} \\
10^{58} \mathrm{ph} / \mathrm{s}\end{array}$ \\
\hline 070529 & 25.7 & 1.43 & 2.5 & 20.70 & $3.8 \mathrm{E}+1$ & $2.1 \mathrm{E}+0$ \\
\hline 070611 & 3.91 & 0.82 & 2.04 & 16.13 & $4.0 \mathrm{E}+0$ & $8.4 \mathrm{E}-1$ \\
\hline 070612A & 106 & 1.51 & 0.617 & 3.65 & $1.0 \mathrm{E}+1$ & $1.5 \mathrm{E}-1$ \\
\hline 070714B & 7.2 & 2.7 & 0.92 & 5.98 & $1.6 \mathrm{E}+0$ & $6.0 \mathrm{E}-1$ \\
\hline 070721B & 36 & 1.5 & 3.626 & 32.48 & $9.8 \mathrm{E}+1$ & $4.1 \mathrm{E}+0$ \\
\hline 070802 & 2.8 & 0.4 & 2.45 & 20.20 & $4.0 \mathrm{E}+0$ & $5.7 \mathrm{E}-1$ \\
\hline 070810A & 6.9 & 1.9 & 2.17 & 17.40 & $7.9 \mathrm{E}+0$ & $2.2 \mathrm{E}+0$ \\
\hline 071003 & 83 & 6.3 & 1.1 & 7.47 & $2.6 \mathrm{E}+1$ & $2.0 \mathrm{E}+0$ \\
\hline 071010A & 2 & 0.8 & 0.98 & 6.47 & $5.1 \mathrm{E}-1$ & $2.0 \mathrm{E}-1$ \\
\hline 071010B & 44 & 7.7 & 0.947 & 6.20 & $1.0 \mathrm{E}+1$ & $1.8 \mathrm{E}+0$ \\
\hline 071021 & 13 & 0.7 & 5.0 & 47.61 & $5.9 \mathrm{E}+1$ & $3.2 \mathrm{E}+0$ \\
\hline 071031 & 9 & 0.5 & 2.692 & 22.66 & $1.5 \mathrm{E}+1$ & $8.3 E-1$ \\
\hline 071112C & 30 & 8 & 0.823 & 5.20 & $5.3 \mathrm{E}+0$ & $1.4 \mathrm{E}+0$ \\
\hline 071117 & 24 & 11.3 & 1.331 & 9.48 & $1.1 \mathrm{E}+1$ & $5.2 \mathrm{E}+0$ \\
\hline 071122 & 5.8 & 0.4 & 1.14 & 7.82 & $2.0 \mathrm{E}+0$ & $1.4 \mathrm{E}-1$ \\
\hline 080210 & 18 & 1.6 & 2.641 & 22.13 & $2.9 \mathrm{E}+1$ & $2.6 \mathrm{E}+0$ \\
\hline 080310 & 23 & 1.3 & 2.426 & 19.95 & $3.2 \mathrm{E}+1$ & $1.8 \mathrm{E}+0$ \\
\hline 080319B & 810 & 24.8 & 0.937 & 6.12 & $1.9 \mathrm{E}+2$ & $5.7 \mathrm{E}+0$ \\
\hline 080319C & 36 & 5.2 & 1.95 & 15.25 & $3.4 \mathrm{E}+1$ & $4.9 \mathrm{E}+0$ \\
\hline 080330 & 3.4 & 0.9 & 1.51 & 11.10 & $2.0 \mathrm{E}+0$ & $5.3 \mathrm{E}-1$ \\
\hline 080411 & 264 & 43.2 & 1.03 & 6.88 & $7.4 \mathrm{E}+1$ & $1.2 \mathrm{E}+1$ \\
\hline 080413A & 35 & 5.6 & 2.432 & 20.01 & $4.9 \mathrm{E}+1$ & $7.8 \mathrm{E}+0$ \\
\hline 080413B & 32 & 18.7 & 1.1 & 7.47 & $1.0 \mathrm{E}+1$ & $6.0 \mathrm{E}+0$ \\
\hline 080430 & 12 & 2.6 & 0.759 & 4.70 & $1.8 \mathrm{E}+0$ & $3.9 \mathrm{E}-1$ \\
\hline 080603B & 24 & 3.5 & 2.69 & 22.64 & $4.0 \mathrm{E}+1$ & $5.8 \mathrm{E}+0$ \\
\hline 080604 & 8 & 0.4 & 1.416 & 10.25 & $4.2 \mathrm{E}+0$ & $2.1 \mathrm{E}-1$ \\
\hline 080605 & 133 & 19.9 & 1.64 & 12.30 & $9.1 \mathrm{E}+1$ & $1.4 \mathrm{E}+1$ \\
\hline 080607 & 240 & 23.1 & 3.036 & 26.22 & $4.9 \mathrm{E}+2$ & $4.7 \mathrm{E}+1$ \\
\hline 080707 & 5.2 & 1 & 1.23 & 8.59 & $2.1 \mathrm{E}+0$ & $4.0 \mathrm{E}-1$ \\
\hline 080710 & 14 & 1 & 0.845 & 5.38 & $2.6 \mathrm{E}+0$ & $1.9 \mathrm{E}-1$ \\
\hline 080721 & 120 & 20.9 & 2.597 & 21.68 & $1.9 \mathrm{E}+2$ & $3.3 \mathrm{E}+1$ \\
\hline 080804 & 36 & 3.1 & 2.202 & 17.72 & $4.2 \mathrm{E}+1$ & $3.6 \mathrm{E}+0$ \\
\hline 080805 & 25 & 1.1 & 1.505 & 11.06 & $1.5 \mathrm{E}+1$ & $6.4 \mathrm{E}-1$ \\
\hline 080810 & 46 & 2 & 3.35 & 29.53 & $1.1 \mathrm{E}+2$ & $4.8 \mathrm{E}+0$ \\
\hline 080905B & 18 & 0.5 & 2.374 & 19.43 & $2.4 \mathrm{E}+1$ & $6.7 \mathrm{E}-1$ \\
\hline 080906 & 35 & 1 & 2 & 15.74 & $3.5 \mathrm{E}+1$ & $9.9 \mathrm{E}-1$ \\
\hline 080916A & 40 & 2.7 & 0.689 & 4.18 & $4.9 \mathrm{E}+0$ & $3.3 E-1$ \\
\hline 080928 & 25 & 2.1 & 1.691 & 12.78 & $1.8 \mathrm{E}+1$ & $1.5 \mathrm{E}+0$ \\
\hline 081007 & 7.1 & 2.6 & 0.53 & 3.03 & $5.1 \mathrm{E}-1$ & $1.9 \mathrm{E}-1$ \\
\hline 081008 & 43 & 1.3 & 1.968 & 15.42 & $4.1 \mathrm{E}+1$ & $1.2 \mathrm{E}+0$ \\
\hline 081028A & 37 & 0.5 & 3.038 & 26.24 & $7.6 \mathrm{E}+1$ & $1.0 \mathrm{E}+0$ \\
\hline 081029 & 21 & 0.5 & 3.847 & 34.88 & $6.3 \mathrm{E}+1$ & $1.5 \mathrm{E}+0$ \\
\hline 081118 & 12 & 0.6 & 2.58 & 21.51 & $1.9 \mathrm{E}+1$ & $9.3 \mathrm{E}-1$ \\
\hline 081121 & 41 & 4.4 & 2.512 & 20.82 & $6.1 \mathrm{E}+1$ & $6.5 \mathrm{E}+0$ \\
\hline 081203A & 77 & 2.9 & 2.1 & 16.71 & $8.3 \mathrm{E}+1$ & $3.1 \mathrm{E}+0$ \\
\hline 081222 & 48 & 7.7 & 2.747 & 23.22 & $8.3 \mathrm{E}+1$ & $1.3 \mathrm{E}+1$ \\
\hline 090102 & 0.68 & 5.5 & 1.548 & 11.45 & $4.2 \mathrm{E}-1$ & $3.4 \mathrm{E}+0$ \\
\hline 090313 & 14 & 0.8 & 3.374 & 29.79 & $3.4 \mathrm{E}+1$ & $1.9 \mathrm{E}+0$ \\
\hline 090418A & 46 & 1.9 & 1.608 & 12.01 & $3.0 \mathrm{E}+1$ & $1.3 \mathrm{E}+0$ \\
\hline 090424 & 210 & 71 & 0.544 & 3.13 & $1.6 \mathrm{E}+1$ & $5.4 \mathrm{E}+0$ \\
\hline 090516A & 90 & 1.6 & 4.105 & 37.68 & $3.0 \mathrm{E}+2$ & $5.3 \mathrm{E}+0$ \\
\hline 090519 & 12 & 0.6 & 3.87 & 35.12 & $3.6 \mathrm{E}+1$ & $1.8 \mathrm{E}+0$ \\
\hline 090529 & 6.8 & 0.4 & 2.62 & 21.97 & $1.1 \mathrm{E}+1$ & $6.4 \mathrm{E}-1$ \\
\hline 090618 & 1050 & 38.9 & 0.54 & 3.10 & $7.8 \mathrm{E}+1$ & $2.9 \mathrm{E}+0$ \\
\hline 090715B & 57 & 3.8 & 3 & 25.85 & $1.1 \mathrm{E}+2$ & $7.6 \mathrm{E}+0$ \\
\hline 090726 & 8.6 & 0.7 & 2.71 & 22.84 & $1.4 \mathrm{E}+1$ & $1.2 \mathrm{E}+0$ \\
\hline 090812 & 58 & 3.6 & 2.452 & 20.22 & $8.2 \mathrm{E}+1$ & $5.1 \mathrm{E}+0$ \\
\hline 090926B & 73 & 3.2 & 1.24 & 8.68 & $2.9 \mathrm{E}+1$ & $1.3 \mathrm{E}+0$ \\
\hline 091018 & 14 & 10.3 & 0.971 & 6.40 & $3.5 \mathrm{E}+0$ & $2.6 \mathrm{E}+0$ \\
\hline 091020 & 37 & 4.2 & 1.71 & 12.96 & $2.7 \mathrm{E}+1$ & $3.1 \mathrm{E}+0$ \\
\hline 091024 & 61 & 2 & 1.092 & 7.40 & $1.9 \mathrm{E}+1$ & $6.3 E-1$ \\
\hline 091029 & 24 & 1.8 & 2.752 & 23.28 & $4.1 \mathrm{E}+1$ & $3.1 \mathrm{E}+0$ \\
\hline 091109A & 16 & 1.3 & 3.288 & 28.88 & $3.7 \mathrm{E}+1$ & $3.0 \mathrm{E}+0$ \\
\hline 091127 & 90 & 46.5 & 0.49 & 2.75 & $5.5 \mathrm{E}+0$ & $2.8 \mathrm{E}+0$ \\
\hline 091208B & 33 & 15.2 & 1.063 & 7.16 & $9.8 \mathrm{E}+0$ & $4.5 \mathrm{E}+0$ \\
\hline
\end{tabular}


A. Mészáros et al.: Cosmological and gamma-ray bursts
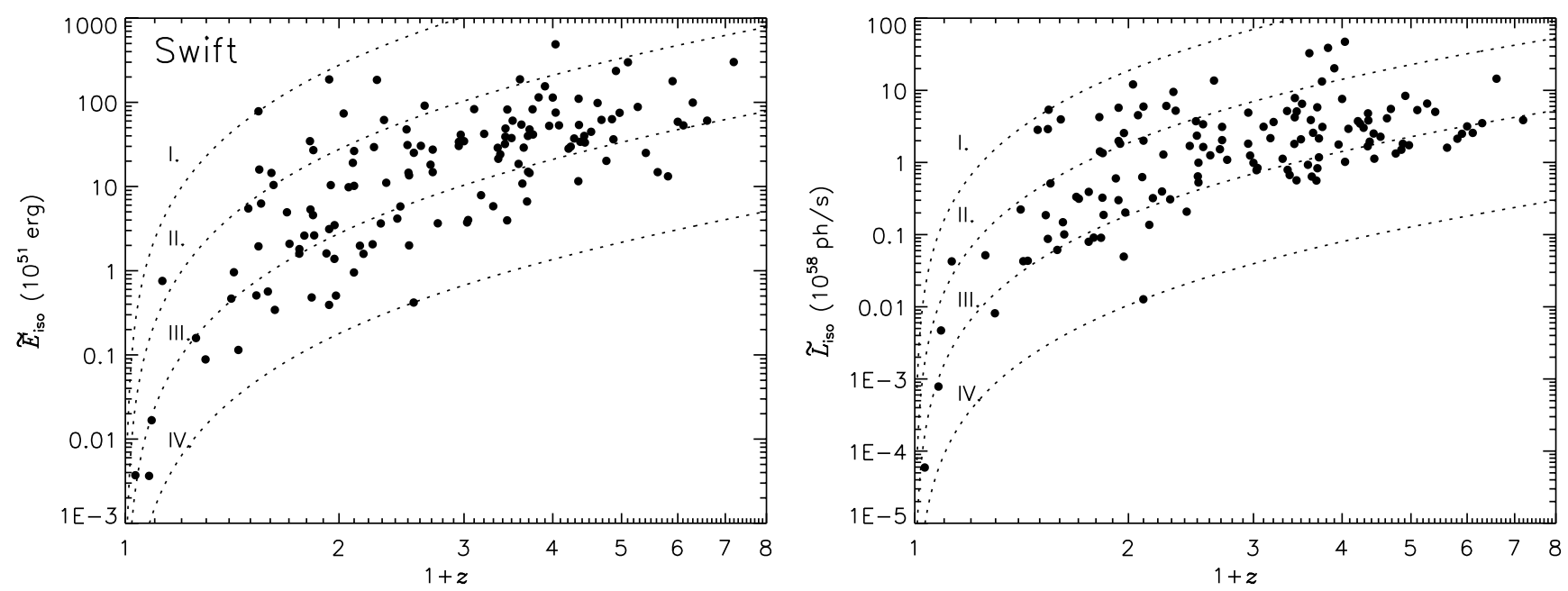

Fig. 4. Left panel: $\tilde{E}_{\text {iso }}$ Vs. $(1+z)\left(\right.$ dots). Dashed contours denote constant fluences (in units $\left.10^{-7} \mathrm{erg} / \mathrm{cm}^{2}\right)$ : I. the maximal fluence, i.e. 1050; II. 105; III. 10.5; IV. the minimal fluence, i.e. 0.68. Right panel: $\tilde{L}_{\text {iso }}$ vs. $(1+z)$ (dots). Dashed contours denote constant peak-fluxes $\left(\right.$ in units ph $\left.\mathrm{cm}^{-2} \mathrm{~s}^{-1}\right)$ : I. the maximal peak-flux, i.e. 71; II. 7.1; III. 0.71; IV. the minimal peak-flux, i.e. 0.04. The objects below a curve at smaller redshifts together with those at higher redshifts and above the curve illustrate the inverse behaviour.

Table 1. continued.

\begin{tabular}{lcccccc}
\hline \hline GRB & $\begin{array}{c}\text { Fluence } \\
10^{-7} \mathrm{erg} / \mathrm{cm}^{2}\end{array}$ & $\begin{array}{c}\text { Peak-flux } \\
\mathrm{ph} /\left(\mathrm{cm}^{2} \mathrm{~s}\right)\end{array}$ & $z$ & $\begin{array}{c}d_{1} \\
\mathrm{Gpc}\end{array}$ & $\begin{array}{c}\tilde{E}_{\text {iso }} \\
10^{51} \mathrm{erg}\end{array}$ & $\begin{array}{c}\tilde{L}_{\text {iso }} \\
10^{58} \mathrm{ph} / \mathrm{s}\end{array}$ \\
\hline $100219 \mathrm{~A}$ & 3.7 & 0.4 & 4.622 & 43.38 & $1.5 \mathrm{E}+1$ & $1.6 \mathrm{E}+0$ \\
$100302 \mathrm{~A}$ & 3.1 & 0.5 & 4.813 & 45.51 & $1.3 \mathrm{E}+1$ & $2.1 \mathrm{E}+0$ \\
$100418 \mathrm{~A}$ & 3.4 & 1 & 0.624 & 3.70 & $3.4 \mathrm{E}-1$ & $1.0 \mathrm{E}-1$ \\
$100425 \mathrm{~A}$ & 4.7 & 1.4 & 1.755 & 13.38 & $3.7 \mathrm{E}+0$ & $1.1 \mathrm{E}+0$ \\
\hline
\end{tabular}

Table 2. BATSE sample with known redshifts.

\begin{tabular}{cccccccc}
\hline \hline GRB & $\begin{array}{c}\text { BATSE } \\
\text { trigger }\end{array}$ & $z$ & $\begin{array}{c}F_{3} \\
10^{-6} \mathrm{erg} / \mathrm{cm}^{2}\end{array}$ & $\begin{array}{c}P_{256} \\
\mathrm{ph} /\left(\mathrm{cm}^{2} \mathrm{~s}\right)\end{array}$ & $\begin{array}{c}d_{1} \\
\mathrm{Gpc}\end{array}$ & $\begin{array}{c}\tilde{E}_{\text {iso }} \\
10^{51} \mathrm{erg}\end{array}$ & $\begin{array}{c}\tilde{L}_{\text {iso }} \\
10^{58} \mathrm{ph} / \mathrm{s}\end{array}$ \\
\hline 970508 & 6225 & 0.835 & 0.88 & 1.18 & 5.3 & $1.6 \mathrm{E}+0$ & $2.2 \mathrm{E}-1$ \\
971214 & 6533 & 3.42 & 4.96 & 2.32 & 30.3 & $1.2 \mathrm{E}+2$ & $5.8 \mathrm{E}+0$ \\
980425 & 6707 & 0.0085 & 1.67 & 1.08 & 0.036 & $2.6 \mathrm{E}-4$ & $1.7 \mathrm{E}-5$ \\
980703 & 6891 & 0.966 & 14.6 & 2.59 & 6.35 & $3.6 \mathrm{E}+1$ & $6.4 \mathrm{E}-1$ \\
990123 & 7343 & 1.600 & 87.2 & 16.63 & 11.9 & $5.7 \mathrm{E}+2$ & $1.1 \mathrm{E}+1$ \\
990506 & 7549 & 1.307 & 51.6 & 22.16 & 9.3 & $2.3 \mathrm{E}+2$ & $9.9 \mathrm{E}+0$ \\
990510 & 7560 & 1.619 & 8.0 & 10.19 & 12.1 & $5.4 \mathrm{E}+1$ & $6.8 \mathrm{E}+0$ \\
991216 & 7906 & 1.02 & 63.7 & 82.10 & 6.8 & $1.7 \mathrm{E}+2$ & $2.3 \mathrm{E}+1$ \\
\hline
\end{tabular}

peak-fluxes are shown after substituting $\tilde{L}_{\text {iso }}={\tilde{L_{0}}}_{(1+z)^{q} \text { and }}$ $\tilde{E}_{\text {iso }}=\tilde{E}_{\mathrm{o}}(1+z)^{q}$ where $\tilde{L}_{\mathrm{o}}$ and $\tilde{E}_{\mathrm{o}}$ are constants, and $q=0 ; 1 ; 2$. The inverse behaviour is quite obvious for $q>1$ and roughly for $z>2$.

The same effect can be similarly illustrated also in Fig. 4 showing the relation $\tilde{E}_{\text {iso }}$ vs. $(1+z)$, and the relation $\tilde{L}_{\text {iso }}$ vs. $(1+z)$. They were calculated again for $H_{0}=71 \mathrm{~km} /(\mathrm{s} \mathrm{Mpc})$, $\Omega_{\mathrm{M}}=0.27$ and $\Omega_{\Lambda}=0.73$. In the figure the curves of constant observable peak-fluxes and fluences are also shown. These curves discriminate the bursts of lower/higher measured values. GRBs below a curve at smaller redshifts are representing the inverse behaviour with respect to those at higher redshifts and above the curve.

\subsection{BATSE sample with known redshifts}

There are 11 bursts, which were observed by BATSE during the period 1997-2000 and for which there are observed redshifts from the afterglow data. For one of them, GRB980329 (BATSE trigger 6665), the redshift has only an upper limit $(z<3.5)$, and hence will not be used here. Two cases (GRB970828 [6350] $z=0.9578$ and GRB000131 [7975] $z=4.5$ ) have determined redshifts, but they have no measured fluences and peak-fluxes, hence they are also excluded. There are remaining $8 \mathrm{GRBs}$, which are collected in Table 2 (see also Bagoly et al. 2003; and Borgonovo 2004). For the definition of fluence we have chosen the fluence from the third BATSE channel between 100 and $300 \mathrm{keV}\left(F_{3}\right)$. This choice is motivated by the observational fact that these fluences in the BATSE Catalog are usually well measured and correlate with other fluences (Bagoly et al. 1998; Veres et al. 2005).

\subsection{BATSE pseudo-redshifts}

In the choice of a BATSE sample with estimated pseudoredshifts one has to take care, since these redshifts are less reliable than the direct redshifts from AGs. We will use here the pseudo-redshifts based on the luminosity-lag relation, restricted 

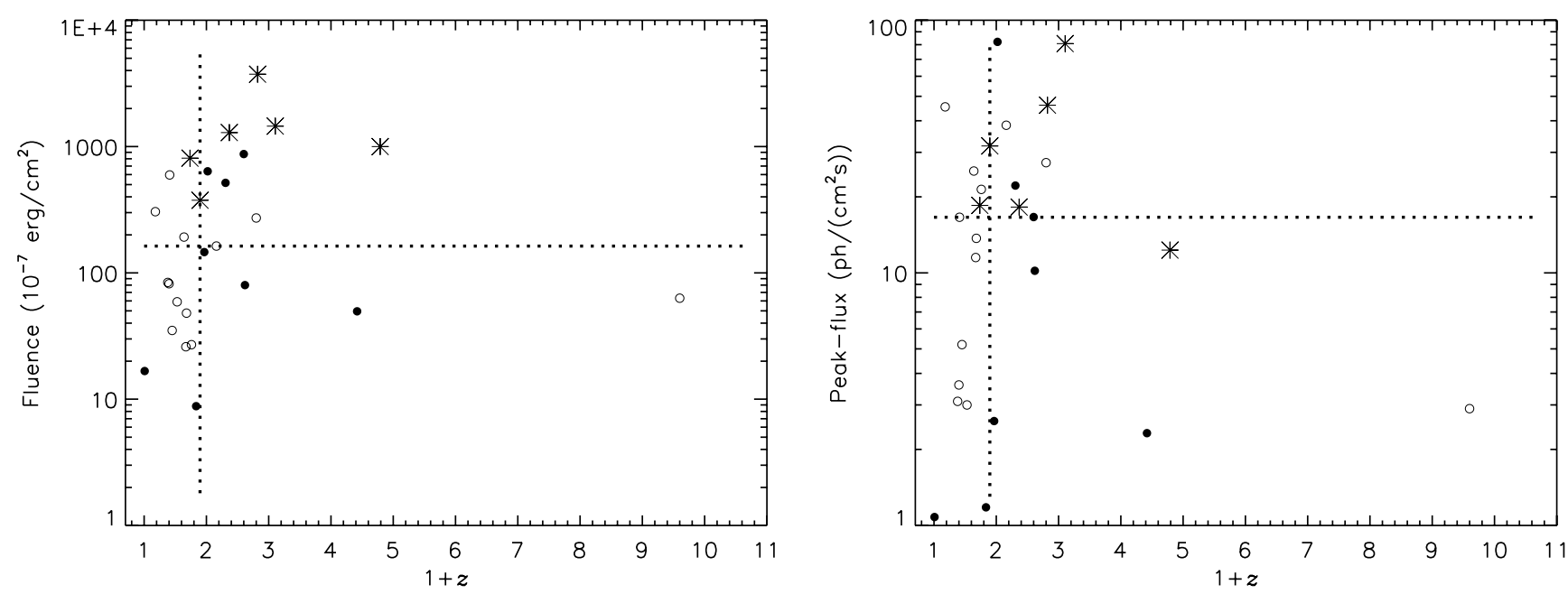

Fig. 5. Distribution of the fluences (left panel) and peak-fluxes (right panel) of the GRBs with known redshifts, where the Fermi GRBs are denoted by asterisks, BATSE GRBs with determined redshifts (pseudo-redshifts) are denoted by dots (circles). The medians separate the area into four quadrants. The objects in the upper right quadrant are brighter and have larger redshifts than the that of GRBs in the lower left quadrant.

Table 3. BATSE sample with pseudo-redshifts.

\begin{tabular}{cccccccc}
\hline \hline GRB & $\begin{array}{c}\text { BATSE } \\
\text { trigger }\end{array}$ & $z$ & $\begin{array}{c}F_{3} \\
10^{-6} \mathrm{erg} / \mathrm{cm}^{2}\end{array}$ & $\begin{array}{c}P_{256} \\
\mathrm{ph} /\left(\mathrm{cm}^{2} \mathrm{~s}\right)\end{array}$ & $\begin{array}{c}d_{1} \\
\mathrm{Gpc}\end{array}$ & $\begin{array}{c}\tilde{E}_{\text {iso }} \\
10^{51} \mathrm{erg}\end{array}$ & $\begin{array}{c}\tilde{L}_{\text {iso }} \\
10^{58} \mathrm{ph} / \mathrm{s}\end{array}$ \\
\hline 911016 & 907 & 0.40 & 8.2 & 3.6 & 2.2 & $3.4 \mathrm{E}+0$ & $1.5 \mathrm{E}-1$ \\
911104 & 999 & 0.67 & 2.6 & 11.5 & 4.1 & $3.1 \mathrm{E}+0$ & $1.4 \mathrm{E}+0$ \\
920525 & 1625 & 1.8 & 27.2 & 27.3 & 13.8 & $2.2 \mathrm{E}+2$ & $2.3 \mathrm{E}+1$ \\
920830 & 1883 & 0.45 & 3.5 & 5.2 & 2.5 & $1.8 \mathrm{E}+0$ & $2.7 \mathrm{E}-1$ \\
921207 & 2083 & 0.18 & 30.5 & 45.4 & 0.86 & $2.3 \mathrm{E}+0$ & $3.4 \mathrm{E}-1$ \\
930201 & 2156 & 0.41 & 59.6 & 16.6 & 2.2 & $2.5 \mathrm{E}+1$ & $6.6 \mathrm{E}-1$ \\
941026 & 3257 & 0.38 & 8.4 & 3.1 & 2.1 & $3.2 \mathrm{E}+0$ & $1.2 \mathrm{E}-1$ \\
950818 & 3765 & 0.64 & 19.2 & 25.3 & 3.8 & $2.0 \mathrm{E}+1$ & $2.7 \mathrm{E}+0$ \\
951102 & 3891 & 0.68 & 4.8 & 13.7 & 4.1 & $5.8 \mathrm{E}+0$ & $1.6 \mathrm{E}+0$ \\
960530 & 5478 & 0.53 & 5.9 & 3.0 & 3.0 & $4.2 \mathrm{E}+0$ & $2.1 \mathrm{E}-1$ \\
960804 & 5563 & 0.76 & 2.7 & 21.4 & 4.7 & $4.1 \mathrm{E}+0$ & $3.2 \mathrm{E}+0$ \\
980125 & 6581 & 1.16 & 16.3 & 38.4 & 8.0 & $5.8 \mathrm{E}+1$ & $1.4 \mathrm{E}+1$ \\
990102 & 7293 & 8.6 & 6.3 & 2.9 & 89.4 & $6.3 \mathrm{E}+2$ & $2.8 \mathrm{E}+1$ \\
\hline
\end{tabular}

Table 4. Fermi sample with known redshifts.

\begin{tabular}{lcccccc}
\hline \hline GRB & $\begin{array}{c}\text { Fluence } \\
10^{-7} \mathrm{erg} / \mathrm{cm}^{2}\end{array}$ & $\begin{array}{c}\text { Peak-flux } \\
\mathrm{ph} /\left(\mathrm{cm}^{2} \mathrm{~s}\right)\end{array}$ & $z$ & $\begin{array}{c}d_{1} \\
\mathrm{Gpc}\end{array}$ & $\begin{array}{c}\tilde{E}_{\text {iso }} \\
10^{51} \mathrm{erg}\end{array}$ & $\begin{array}{c}\tilde{L}_{\text {iso }} \\
10^{58} \mathrm{ph} / \mathrm{s}\end{array}$ \\
\hline 090323 & 1000 & 12.3 & 3.79 & 31.88 & $2.66 \mathrm{E}+3$ & $3.27 \mathrm{E}+1$ \\
090328 & 809 & 18.5 & 0.736 & 4.53 & $1.34 \mathrm{E}+2$ & $2.62 \mathrm{E}+0$ \\
$090902 \mathrm{~B}$ & 3740 & 46.1 & 1.822 & 14.02 & $3.12 \mathrm{E}+3$ & $3.84 \mathrm{E}+1$ \\
$090926 \mathrm{~A}$ & 1450 & 80.8 & 2.106 & 16.77 & $1.57 \mathrm{E}+3$ & $8.76 \mathrm{E}+1$ \\
091003 & 376 & 31.8 & 0.897 & 5.79 & $7.96 \mathrm{E}+1$ & $6.73 \mathrm{E}+0$ \\
$100414 \mathrm{~A}$ & 1290 & 18.22 & 1.368 & 9.81 & $6.28 \mathrm{E}+2$ & $8.87 \mathrm{E}+0$ \\
\hline
\end{tabular}

to the sample in Ryde et al. (2005), where also the spectroscopic studies support the estimations. In Table 3 we collect 13 GRBs using Table 3 of Ryde et al. (2005). We do not consider two GRBs (BATSE triggers 973 and 3648) from Ryde et al. (2005), since their estimated pseudo-redshifts are ambiguous.

\subsection{Fermi sample}

The Fermi sample contains only 6 GRBs with known redshifts together with peak-fluxes and fluences (Bissaldi \& Connaughton 2009; Bissaldi 2009; van der Horst et al. 2009; Rau et al. 2009,b; Foley et al. 2010). They are collected in Table 4. The peak-fluxes and fluences were measured over the energy range $50 \mathrm{keV}-10 \mathrm{MeV}$ for GRB090902B and in the range $8 \mathrm{keV}-1 \mathrm{MeV}$ for the remaining five objects.

\subsection{Inversion in the BATSE and Fermi samples}

The fluence (peak-flux) vs. redshift relationship of the Fermi and of the two BATSE samples are summarized in Fig. 5. For demonstrating the inversion effect - similarly to the case of the Swift sample - the medians also marked with dashed lines. Here it is quite evident that some of the distant bursts exceed in their observed fluence and peak-fluxes those of having smaller redshifts. Here again the GRBs in the upper right quadrants are apparently brighter than those in the lower left one, although their redshifts 
are larger. Note that in the upper right quadrants are even more populated than the lower right quadrants. In other words, here the trend of the increasing of peak-flux (fluence) with redshift is evident, and the assumption of the Sect. 2.2. need not be used.

\section{Results and discussion}

It follows from the previous section that in all samples both for the fluences and for the peak-fluxes the "inverse" behaviour, discussed in Sect. 2, can happen. The answer on the question of the title of this article is therefore that "this does not need to be the case". Simply, the apparently faintest GRBs need not be also the most distant ones. This is in essence the key result of this article.

It is essential to note that in this paper no assumptions were made about the models of the long GRBs. Also the cosmological parameters did not need to be specified.

All this means that faint bursts in the BATSE Catalog simply need not be at larger redshifts, because the key "natural" assumption - apparently fainter GRBs should on average be at higher redshifts - does not hold. All this also means that the controversy about the fraction of GRBs at very high redshifts in BATSE Catalog may well be overcame: no large fraction of GRBs needs to be at very high redshifts, and the redshift distribution of long GRBs - coming from the Swift sample - may well occur also for the BATSE sample. Of course, this does not mean that no GRBs can be at, say, $8.3<z<20$. As proposed first by Mészáros \& Mészáros (1996), at these very high redshifts GRBs may well occur, but should give a minority (say $10 \%$ or so) in the BATSE Catalog similarly the Swift sample. This point of view is supported by newer studies, too (Bromm \& Loeb 2006; Jakobsson et al. 2006; Gehrels et al. 2009; Butler et al. 2010).

At the end it is useful to raise again that the purpose of this paper was not to study the intrinsic evolution of the luminosities $L(z)$ from the energy range $E_{1} \leq E \leq E_{2}$. To carry out such study, one should consider three additional reasons that may be responsible for the growth of the average value of $\tilde{L}_{\text {iso }}$ with $(1+z)$ : 1) K-correction; 2) the beaming; 3) selection biases due to the instrument's threshold and other instrumental effects. For example, on Fig. 4 the main parts in the right-bottom sections below the curves IV - corresponding to the values below the instrumental thresholds in fluence/peak-flux - are not observable. Nonetheless, even using these biased data, the theoretical considerations stated in the Sect. 2 and conclusions of the next sections are valid.

\section{Conclusions}

The results of this paper can be summarized as follows:

1. the theoretical study of the $z$-dependence of the observed fluences and peak-fluxes of GRBs have shown that fainter bursts could well have smaller redshifts;

2. this is really fulfilled for the four different samples of long GRBs;

3. these results do not depend on the cosmological parameters and on the GRB models;

4. all this suggests that the estimations, leading to a large fraction of BATSE bursts at $z>5$, need not be correct.

Acknowledgements. We wish to thank Z. Bagoly, L. G. Balázs, I. Horváth, S. Larsson, P. Mészáros and P. Veres for useful discussions and comments on the manuscript. The useful remarks of the anonymous referee are kindly acknowledged. This study was supported by the OTKA grant K77795, by the Grant Agency of the Czech Republic grants No. 205/08/H005, and P209/10/0734, by the project SVV 261301 of the Charles University in Prague, by the Research Program MSM0021620860 of the Ministry of Education of the Czech Republic, and by the Swedish National Space Agency.

\section{References}

Amati, L., Frontera, F., Tavani, M., et al. 2002, A\&A, 390, 81

Atteia, J.-L. 2003, A\&A, 407, L1

Bagoly, Z., \& Veres, P. 2009a, Gamma-ray burst: Sixth Huntsville Symp., AIPC 1133,473

Bagoly, Z., \& Veres, P. 2009b, Baltic Astron., 18, 297

Bagoly, Z., Mészáros, A., Horváth, I., Balázs, L. G., \& Mészáros, P. 1998, ApJ, 498, 342

Bagoly, Z., Csabai, I., Mészáros, A., et al. 2003, A\&A, 398, 919

Bagoly, Z. Mészáros, A., Balázs, L. G., et al. 2006, A\&A, 453, 797

Balázs, L. G., Bagoly, Z., Horváth, I., Mészáros, A., \& Mészáros, P. 2003, A\&A, 401, 129

Band, D., Norris, J. P., \& Bonnell, J. T. 2004, ApJ, 613, 484

Bissaldi, E. 2009, GCN, 9933, 1

Bissaldi, E., \& Connaughton, V. 2009, GCN, 9866, 1

Borgonovo, L. 2004, A\&A, 418, 487

Bromm, V., \& Loeb, A. 2006, ApJ, 642, 382

Butler, N. R., Bloom, J. S., \& Poznanski, D. 2010, ApJ, 711, 495

Carroll, S. M., Press, W. H., \& Turner, E. L. 1992, ARA\&A, 30, 499

Foley, S., et al. 2010, GCN, 10595

Gehrels, N., Ramirez-Ruiz, E., \& Fox, D. B. 2009, ARA\&A, 47, 567

Ghirlanda, G., Ghisellini, G., \& Firmani, C. 2005, MNRAS, 361, L10

Guidorzi, C., Frontera, F., Montanari, E., et al. 2005, MNRAS, 363, 315

Hakkila, J., Giblin, T. W., Roiger, R. J., et al. 2003, ApJ, 582, 320

Horváth, I. 1998, ApJ, 508, 757

Horváth, I. 2002, A\&A, 392, 791

Horváth, I., Mészáros, P., \& Mészáros, A. 1996, ApJ, 470, 56

Horváth, I., Balázs, L. G., Bagoly, Z., \& Veres, P. 2008, A\&A, 489, L1

Horváth, I., Bagoly, Z., Balázs, L. G., Tusnády, G., \& Veres, P. 2009, Fermi Symposium, eConf Proc. C0911022 [arXiv: 0912 . 3724]

Jakobsson, P., Levan, A., Fynbo, J. P. U., et al. 2006, A\&A, 447, 897

Lamb, D. Q., \& Reichart, D. E. 2000, ApJ, 536, 1

Lee, A., Bloom, E. D., \& Petrosian, V. 2000, ApJS, 131, 21

Lin, J. R., Zhang, S. N., \& Li, T. P. 2004, ApJ, 605, 819

Lloyd-Ronning, N. M., Fryer, C. L., \& Ramirez-Ruiz, E. 2002, ApJ, 574, 554

Mészáros, P. 2006, Rep. Progr. Phys., 69, 2259

Mészáros, P., \& Mészáros, A. 1995, ApJ, 449, 9

Mészáros, A., \& Mészáros, P. 1996, ApJ, 466, 29

Mészáros, A., Bagoly, Z., Balázs, L. G., \& Horváth, I. 2006, A\&A, 455, 785

Mészáros, A., Balázs, L. G., Bagoly, Z., \& Veres, P. 2009, Gamma-ray bursts: Sixth Huntsville Symp., AIP Conf. Proc., 1133, 483

Norris, J. P. 2002, ApJ, 579, 386

Norris, J. P. 2004 Baltic Astron., 13, 221

Norris, J. P., Marani, G. F., \& Bonnell, J. T. 2000, ApJ, 534, 248

Norris, J. P., Scargle, J. D., \& Bonnell, J. T. 2001, Gamma-Ray Bursts in the Afterglow Era, ed. E. Costa, F. Frontera, \& J. Hjorth (Springer-Verlag), 40 Paczyński, B. 1992, Nature, 355, 521

Petrosian, V., Bouvier, A., \& Ryde, F. 2009 [arXiv: 0909. 5051]

Piran, T. 2004, Rev. Mod. Phys., 76, 1143

Ramirez-Ruiz, E., \& Fenimore, E. E. 2000, ApJ, 539, 712

Rau, A. 2009, GCN, 9983, 1

Rau, A., Connaughton, V., \& Briggs, M. 2009, GCN, 9057, 1

Reichart, D. E., \& Mészáros, P. 1997, ApJ, 483, 597

Reichart, D. E., Lamb, D. Q., Fenimore, E. E., et al. 2001, ApJ, 552, 57

Ryde, F., Kocevski, D., Bagoly, Z., Ryde, N., \& Mészáros, A. 2005, A\&A, 432, 105

Schaefer, B. E. 2003, ApJ, 583, L67

Schaefer, B. E., Deng, M., \& Band, D. L. 2001, ApJ, 563, L123

Tonry, J. L., Schmidt, B. P., Barris, B., et al. 2003, ApJ, 594, 1

van der Horst, A. J., \& Xin, L. P. 2009, GCN, 9035

Varga, B., Horváth, I., Balázs, L. G., et al. 2005, Nuovo Cim. C., 28, 861

Vavrek, R., Balázs, L. G., Mészáros, A., Horváth, I., \& Bagoly, Z. 2008, MNRAS, 391, 1741

Veres, P., Babayev, A. M., Artunç, E., \& Cakmak, S. 2005, Nuovo Cim. C., 28, 355

Weinberg, S. 1972, Gravitation and Cosmology (New York: J. Wiley and Sons.) Wolf C., \& Podsiadlowski P. 2007, MNRAS, 375, 1049

Zhang, B., Zhang, B.-B., Virgili, F. J., et al. 2009, ApJ, 703, 1696 